\title{
Synthesis of novel nanostructured chiral poly(amide-imide)s containing dopamine and natural amino acids
}

\author{
SHADPOUR MALLAKPOUR ${ }^{\mathrm{a}, \mathrm{b}, *}$ and AMIN ZADEHNAZARI ${ }^{\mathrm{a}}$ \\ ${ }^{a}$ Organic Polymer Chemistry Research Laboratory, Department of Chemistry, \\ ${ }^{b}$ Nanotechnology and Advanced Materials Institute, Isfahan University of Technology, \\ Isfahan, 84156-83111, Islamic Republic of Iran \\ e-mail:mallak@cc.iut.ac.ir; mallakpour84@alumni.ufl.edu; mallak777@yahoo.com
}

MS received 8 January 2012; revised 16 March 2012; accepted 25 May 2012

\begin{abstract}
Four new thermally stable and optically active poly(amide-imide)s (PAI)s with good inherent viscosities were synthesized from the direct polycondensation reaction of $\mathrm{N}, \mathrm{N}^{\prime}$-(pyromellitoyl)-bis-L- $\alpha$-amino acids with 3,5-diamino-N-(3,4-dihydroxy-phen-ethyl)benzamide in a medium consisting of a molten salt, tetrabutylammonium bromide, and triphenyl phosphite as the activator. The polymerization reactions produced a series of novel PAIs containing dopamine segment in the side chain in high yield with inherent viscosities between 0.33 and $0.49 \mathrm{dL} / \mathrm{g}$. The obtained polymers were typically characterized by means of FT-IR, ${ }^{1} \mathrm{H}$ NMR spectroscopy, elemental analyses, powder X-ray diffraction, field emission scanning electron microscopy, inherent viscosity, and solubility tests. Thermal properties and flame retardant behaviour of the PAIs were also investigated using thermal gravimetric analysis (TGA and DTG) and limiting oxygen index (LOI). Data obtained by thermal analysis revealed that these polymers showed good thermal stability. Furthermore, high char yield in TGA and good LOI values indicated that the obtained polymers were capable of exhibiting good flame retardant properties.
\end{abstract}

Keywords. Thermally stable; green chemistry; optically active polymers; tetrabutylammonium bromide.

\section{Introduction}

Polymers play an important role in the development of materials for industrial applications such as biochemical science and in the pharmaceutical industry due to their exceptional properties. ${ }^{1}$ They are relatively inexpensive, can be functionalized to achieve required optical, electronic, or mechanical properties, and have demonstrated compatibility with various patterning methods. They can be 'nanostructured' since they exhibit pertinent structural features down at the nanometer length scale. Nanostructured polymers are currently gaining world-wide interest, because of their unique properties and promising potential applications in many areas such as biosensors, integrated optics, drug delivery systems, and molecular electronics. ${ }^{2-4}$ There are many ways to produce nanostructured polymers including self-assembling method, LangmuirBlodget technique, layer-by-layer technique, etc. ${ }^{5-7}$

\footnotetext{
*For correspondence
}

In recent years, environmentally-friendly reaction processes have been vigorously studied from the standpoint of green chemistry. Most recently, ionic liquids (IL)s have gained much attention as green reaction solvents for organic synthesis. ${ }^{8,9}$ When an IL is used as a reaction medium, the solute is solvated by ions, where the reaction proceeds under quite different conditions as compared to using water or common organic solvents. ${ }^{10}$ The solvent power of ILs can be tuned by designing the structure of cations and anions and choosing appropriate combinations of them. For example, ILs containing quaternary ammonium cation such as molten tetrabutylammonium bromide (TBAB) salt is not only used as reaction solvent, but also exhibit catalytic properties. ${ }^{11-14}$ This molten ionic salt has very high solubility in water and polar organic solvents. Utilization of this property enables recovery and reuse of it, after extracting the product. This can help to reduce the waste of traditional solvents which are rarely reused. It was also used as reaction medium in several step-growth polymerization reactions. ${ }^{15-17}$

Numerous studies concerned with the synthesis and characterization of optically active polymers have been 
carried out, mainly in the past few decades, because of their chiroptical and stereospecific properties. ${ }^{18}$ They are used as catalysts for asymmetric synthesis, ${ }^{19}$ as a chiral stationary phase (CSP) for enantioselective separation in high performance liquid chromatography (HPLC), ${ }^{20}$ electrodes for enantioselective recognition for performing bioelectro synthesis, ${ }^{21}$ microwave absorbents, ${ }^{22}$ membrane separation technology, ${ }^{23}$ liquid crystals, ${ }^{24}$ and nonlinear optics. ${ }^{25,26}$ A direct and effective way for synthesizing chiral polymers is to introduce chiral elements into the polymer backbone or side chains. Some groups have made large contributions to this significant research field, including Okamoto and co-workers, ${ }^{27}$ Masuda et al.,${ }^{28}$ Yashima et al. ${ }^{29}$ Aoki and co-workers, ${ }^{30}$ etc. In the polycondensation reactions our group uses amino acids as chiral inducting agents. These materials are naturally occurring compounds and synthetic polymers based on amino acids are expected to be biodegradable and biocompatible. In addition to optical properties of these polymers, the solubility of them was improved without significant loss of mechanical and thermal properties. ${ }^{31}$

Based on our previous studies, we describe here the preparation and basic characterization of a series of novel heat resistant and optically active nanostructured poly(amide-imide)s (PAI)s containing amino acid and dopamine moiety in a medium consisting of molten TBAB salt and triphenyl phosphite (TPP) by reaction of 3,5-diamino-N-(3,4-dihydroxyphenethyl)benzamide with four different $\mathrm{N}, \mathrm{N}^{\prime}$-(pyromellitoyl)-bis-L-amino acids.

\section{Experimental}

\subsection{Materials}

All chemicals used in this study were obtained commercially from Fluka Chemical Co. (Switzerland), Aldrich Chemical Co. (Milwaukee, WI) and Merck Chemical Co. (Germany). Dopamine hydrochloride (3hydroxytyraminium chloride), 3,5-dinitrobenzoylchloride, N,N'-dimethylacetamide (DMAc), N-methyl2pyrrolidone (NMP), and propylene oxide from Merck were used for the synthesis of mediators. Propylene oxide was used as acid scavenger. $\mathrm{N}, \mathrm{N}^{\prime}-$ dimethylformamide (DMF) $\left(\mathrm{d}=0.94 \mathrm{~g} \mathrm{~cm}^{-3}\right.$ at $\left.20^{\circ} \mathrm{C}\right)$, and DMAc as solvent $\left(\mathrm{d}=0.94 \mathrm{~g} \mathrm{~cm}^{-3}\right.$ at $\left.20^{\circ} \mathrm{C}\right)$ were distilled over barium oxide under reduce pressure. Other reagents were used without further purification.

\subsection{Techniques}

Proton nuclear magnetic resonance $\left({ }^{1} \mathrm{H}\right.$ NMR, $400 \mathrm{MHz}$ ) spectra were carried out at a Bruker (Germany) Avance 500 instrument at room temperature (RT) in N, $\mathrm{N}^{\prime}$-dimethylsulphoxide- $d_{6}\left(\mathrm{DMSO}-d_{6}\right)$. Multiplicities of proton resonance were designated as singlet (s), doublet (d), triplet (t), and multiplet (m). FT-IR spectra were recorded on a spectrophotometer (Jasco-680, Japan). The spectra of solids were obtained using $\mathrm{KBr}$ pellets. The vibrational transition frequencies are reported in wavenumbers $\left(\mathrm{cm}^{-1}\right)$. Band intensities are assigned as weak (w), medium (m), strong (s), and broad (br). Inherent viscosities were measured by using a Cannon Fenske Routine Viscometer (Germany) at the concentration of $0.5 \mathrm{~g} \mathrm{dL}^{-1}$ at $25^{\circ} \mathrm{C}$. Specific rotations were measured by a Jasco Polarimeter (Japan). Thermal gravimetric analysis (TGA) is performed with a STA503 win TA at a heating rate of

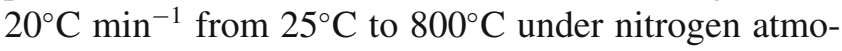
sphere. The X-ray diffractograms of polymers were recorded using an X-ray diffraction (XRD) (Bruker, D8ADVANCE, Germany) with a copper target at $40 \mathrm{kV}$ and $35 \mathrm{~mA}$ and $\mathrm{Cu} \mathrm{K} \alpha \lambda=1.54 \AA$ in the range of $10-80^{\circ}$ at the speed of $0.05^{\circ} \mathrm{min}^{-1}$. The morphology of the polymers was observed using field emission scanning electron microscope (FE-SEM) (HITACHI S4160). The effect of ultrasonic radiation on the size of polymer particles was investigated by MISONIX ultrasonic liquid processors, XL-2000 SERIES. Ultrasound was a wave of frequency $2.25 \times 10^{4} \mathrm{~Hz}$ and power $100 \mathrm{~W}$.

\subsection{Monomer synthesis procedure}

3,5-Diamino-N-(3,4-dihydroxyphenethyl)benzamide (4) as a diamine monomer was prepared according to our published article and is shown in scheme $1 .{ }^{32}$

$\mathrm{N}, \mathrm{N}^{\prime}$-(pyromellitoyl)-bis-L-amino acids $\mathbf{7 a - 7 d}$ were prepared in quantitative yields by the condensation reaction of pyromellitic dianhydride $(1,2,4,5-$ benzene-tetra-carboxylicacid-1,2,4,5-dianhydride) with L-leucine, ${ }^{33}$ L-isoleucine, ${ }^{34} \mathrm{~L}$-valine, ${ }^{35}$ and L-alanine ${ }^{36}$ in acetic acid solution.

\subsection{Polymer synthesis procedure}

The PAIs were prepared by the following procedure: for the synthesis of PAI8a, A mixture of $0.10 \mathrm{~g}(2.25 \times$ $\left.10^{-4} \mathrm{~mol}\right)$ of diacid $7 \mathrm{a}, 0.064 \mathrm{~g}\left(2.25 \times 10^{-4} \mathrm{~mol}\right)$ of diamine 4 and $0.29 \mathrm{~g}$ of TBAB $\left(9.00 \times 10^{-4} \mathrm{~mol}\right)$ was ground until a powder was formed. After the mixture 


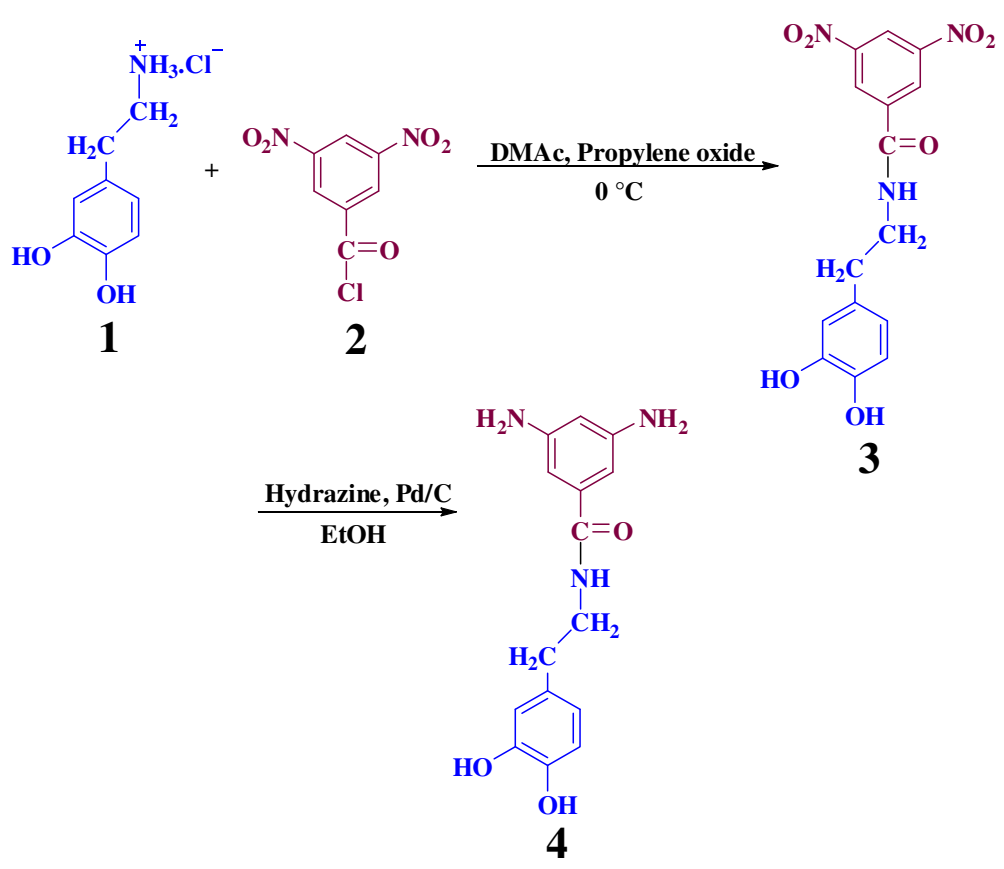

Scheme 1. Synthetic rute of diamine 4 .

was completely ground, it was transferred into a $25 \mathrm{~mL}$, round-bottom flask and then $0.23 \mathrm{~mL}\left(9.00 \times 10^{-4} \mathrm{~mol}\right)$ of TPP was added to the mixture which was heated until a homogeneous solution was formed. Then, the solution was stirred for $12 \mathrm{~h}$ at $120^{\circ} \mathrm{C}$, and the viscous solution was precipitated in $15 \mathrm{~mL}$ of methanol. The yellow solid was filtered off and dried to give $0.168 \mathrm{~g}(92 \%)$ of PAI8a. The other PAIs, PAI8b-PAI8d were prepared by a similar procedure.

PAI8a: FT-IR $\left(\mathrm{KBr}, \mathrm{cm}^{-1}\right): 3,338(\mathrm{~m}, \mathrm{br}, \mathrm{NH}$ and $\mathrm{OH}$ stretching), 3,098 (w, C-H aromatic) 2,960 (m, C$\mathrm{H}$ aliphatic), 2,873 (w, C-H aliphatic), 1,775 (m, C=O imide, asymmetric stretching), 1,722 ( $\mathrm{s}, \mathrm{C}=\mathrm{O}$ imide, symmetric stretching), 1,647 ( $\mathrm{m}, \mathrm{C}=\mathrm{O}$ amide, stertching), 1,599 (s), 1,449 (s), 1,382 (s, CNC axial stretching), 1,116 (m, CNC transverse stretching), 1,078 (w), 960 (m), 879 (m), 821 (m), 726 (s, CNC out-of-plane bending), $691(\mathrm{w})$.

PAI8b: ${ }^{1} \mathrm{H}-\mathrm{NMR}\left(400 \mathrm{MHz}, \mathrm{DMSO}-d_{6}, \mathrm{ppm}\right): 0.92$ (t, $6 \mathrm{H}, \mathrm{CH}_{3}$, distorted), $1.02-1.03\left(\mathrm{~d}, 6 \mathrm{H}, \mathrm{CH}_{3}, J=\right.$ $3.68 \mathrm{~Hz}), 1.52\left(\mathrm{~m}, 4 \mathrm{H}, \mathrm{CH}_{2}\right), 1.70(\mathrm{~m}, 2 \mathrm{H}, \mathrm{CH}), 2.29$ (t, 2H, CH, distorted), 2.68 (t, 2H, CH, distorted), 4.71$4.72(\mathrm{~d}, 2 \mathrm{H}, \mathrm{CH}, J=5.80 \mathrm{~Hz}), 6.42-6.43(\mathrm{~d}, 1 \mathrm{H}, \mathrm{Ar}-\mathrm{H}$, $J=5.92 \mathrm{~Hz}), 6.58(\mathrm{~s}, 1 \mathrm{H}, \mathrm{Ar}-\mathrm{H}), 6.63-6.64(\mathrm{~d}, 1 \mathrm{H}, \mathrm{Ar}-$ $\mathrm{H}, J=6.40 \mathrm{~Hz}), 7.90$ (s, 2H, Ar-H), 8.18 (s, 1H, Ar-H), 8.29 (s, 2H, Ar-H), 8.55 (s, 1H, OH), 8.61 (s, 1H, OH), 8.66 (s, 1H, NH), 10.24 (s, 2H, NH).

Elemental analysis: calculated for $\left(\mathrm{C}_{37} \mathrm{H}_{37} \mathrm{~N}_{5} \mathrm{O}_{9}\right)_{\mathrm{n}}: \mathrm{C}$, $63.88 \%$; H, 5.36\%; N, 10.07\%. Found: C, 63.22\%; H, $5.24 \% ; \mathrm{N}, 10.13 \%$.
PAI8c: FT-IR $\left(\mathrm{KBr}, \mathrm{cm}^{-1}\right): 3,420(\mathrm{~m}, \mathrm{br}, \mathrm{NH}$ and $\mathrm{OH}$ stretching), 3,100 (w, C-H aromatic), 2,964 (w, C$\mathrm{H}$ aliphatic), 2,932 (w, C-H aliphatic), 1,776 (m, C=O imide, asymmetric stretching), 1,722 ( $\mathrm{s}, \mathrm{C}=\mathrm{O}$ imide, symmetric stretching), 1,647 ( $\mathrm{s}, \mathrm{C}=\mathrm{O}$ amide, stertching), 1,599 (s), 1,554 (s), 1,446 (s), 1,382 (s, CNC axial stretching), 1,213 (m, CNC transverse stretching), 1,077 (m), 879 (m), 762 (m), 727 (s, CNC out-of-plane bending), $689(\mathrm{w})$.

Elemental analysis: calculated for $\left(\mathrm{C}_{35} \mathrm{H}_{33} \mathrm{~N}_{5} \mathrm{O}_{9}\right)_{\mathrm{n}}$ : C, $62.96 \%$; H, $4.98 \%$; N, $10.49 \%$. Found: C, $62.46 \%$; H, $5.00 \%$; N, $10.58 \%$.

PAI8d: FT-IR $\left(\mathrm{KBr}, \mathrm{cm}^{-1}\right): 3,409(\mathrm{~m}, \mathrm{br}, \mathrm{NH}$ and OH stretching), 3,100 (w, C-H aromatic), 2,961 (w, C$\mathrm{H}$ aliphatic), 2,932 (w, C-H aliphatic), 1,774 (m, C=O imide, asymmetric stretching), 1,719 ( $\mathrm{s}, \mathrm{C}=\mathrm{O}$ imide, symmetric stretching), 1,647 ( $\mathrm{m}, \mathrm{C}=\mathrm{O}$ amide, stertching), 1,600 (s), 1,559 (m), 1,448 (s), 1,383 (s, CNC axial stretching), 1,152 (m, CNC transverse stretching), 1,076 (m), 879 (m), 764 (m), 727 (s, CNC out-of-plane bending), $690(\mathrm{w})$.

\section{Results and discussion}

\subsection{Monomer synthesis}

Diamine monomer 4 was synthesized by using a two-step reaction according to our previous work 

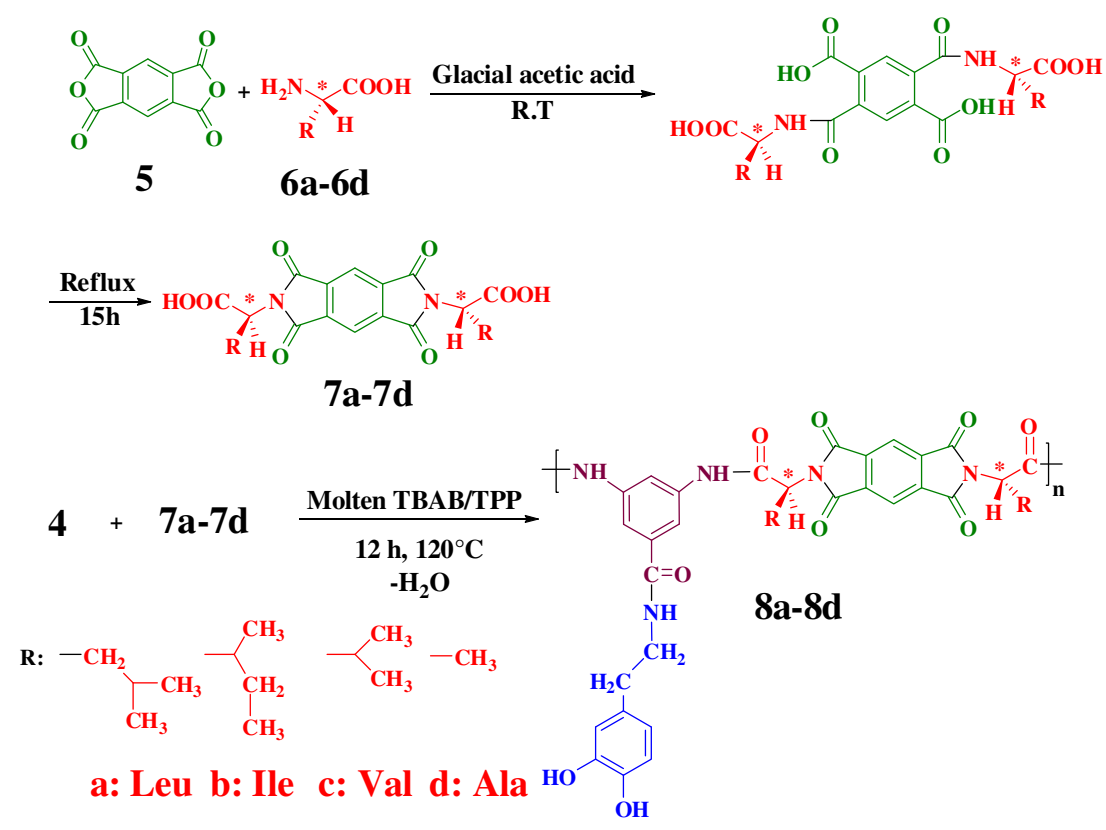

Scheme 2. Synthesis of PAIs $\mathbf{8 a - 8 d}$.

(scheme 1). ${ }^{32}$ Diacid monomers were synthesized by the condensation reaction of an equimolar amount of pyromellitic dianhydride (1) and different amino acids [L-leucine (6a), L-isoleucine (6b), S-valine (6c), and Lalanine (6d)] in refluxing acetic acid solution, as shown in scheme $2 .{ }^{33-36}$

\subsection{Polymer synthesis}

The direct polycondensation of a dicarboxylic acid and diamine in a conventional organic solvent is one of the well-known methods for PAI synthesis. The use of environmentally benign reaction media is very important in view of today's environment-conscious attitudes. The utilization of organic solvents, which are most often used for conventional polymerization media, has numerous disadvantages: they may be flammable, toxic, and volatile, which increases the potential hazard of environmental pollution due to solvent loss. The use of green solvents such as ILs appears to eliminate these drawbacks, while retaining the advantages of common organic solvents or even ensuring better conditions. So, in this research we tried to eliminate common organic solvents such as NMP, DMAc, and DMF and performed the polymerization reaction under green conditions to report a simple, safe, and efficient method for synthesis of several optically active PAIs via direct polycondensation reaction of several natural amino acid-based diacids (7a-7d) with an aromatic dopamine- based diamine (4) in molten TBAB in the presence of TPP (scheme 2). This IL was selected because it proved to be the most valuable among those employed in our recent works. ${ }^{15-17}$ Runs in IL were carried out by thermal heating technique. The entire polycondensation reaction readily proceeds in a homogeneous solution and after stringy work-up tough and precipitates were formed. The syntheses and some physical properties of these new PAIs (8a-8d) are given in table 1 . All the polymers were obtained in high yields (85$92 \%$ ), and the inherent viscosities were $0.33-0.49 \mathrm{dL} / \mathrm{g}$ which were measured in DMF solutions. Also, the resulting polymers showed yellow colour. All of them are optically active, because they have a chiral centre from amino acid residue in their pendant groups. The structure of the PAIs was confirmed by FT-IR and ${ }^{1} \mathrm{H}$ NMR spectroscopy, and elemental analysis technique.

\subsection{Polymer characterization}

The FT-IR spectra of all polymers showed absorptions around $1719-1776 \mathrm{~cm}^{-1}$, commonly attributed to the asymmetric and symmetric stretches of carbonyl groups. The presence of imide heterocycle in these polymers was revealed by absorption around 1382 and $726 \mathrm{~cm}^{-1}$ which belong to carbonyl bendings of imide. Bands of amide $\mathrm{N}-\mathrm{H}$ and hydroxyl groups appeared at around $3338-3420 \mathrm{~cm}^{-1}$. A representative FT-IR spectrum for PAI $8 \mathrm{~b}$ is illustrated in figure 1 . The structure of 
Table 1. Synthesis and some physical properties of PAI8a-PAI8d prepared in molten TBAB.

\begin{tabular}{lcccccc}
\hline Diacid & Polymer $^{\mathrm{a}}$ & Yield $(\%)$ & Inherent viscosity $^{\mathrm{b}}(\mathrm{dL} / \mathrm{g})$ & {$[\alpha]_{N a, 589}^{25, b}(\mathrm{deg} .)^{\mathrm{b}}$} & {$[\alpha]_{H g}^{25}(\mathrm{deg} .)^{\mathrm{c}}$} & Colour \\
\hline 7a & PAI8a & 92 & 0.35 & -30.41 & -18.90 & Yellow \\
7b & PAI8b & 87 & 0.43 & -29.38 & -16.93 & Yellow \\
7c & PAI8c & 88 & 0.49 & -31.12 & -25.46 & Yellow \\
$\mathbf{7 d}$ & PAI8d & 85 & 0.33 & -23.15 & -16.65 & Yellow \\
\hline
\end{tabular}

${ }^{\text {a }}$ Polymers were precipitated in methanol

${ }^{\mathrm{b}}$ Measured at a concentration of $0.5 \mathrm{~g} \mathrm{dL}^{-1}$ in $\mathrm{DMF}$ at $25^{\circ} \mathrm{C}$

${ }^{\mathrm{c}}$ Measured without filter

PAI8b was also confirmed with ${ }^{1} \mathrm{H}$ NMR spectroscopy (figure 2). In the ${ }^{1} \mathrm{H}$ NMR spectrum of this polymer, appearances of the $\mathrm{N}-\mathrm{H}$ protons of amide groups at 8.66 and 10.24 , as two singlet peaks, and $\mathrm{OH}$ groups at 8.55 and $8.61 \mathrm{ppm}$ as two singlet peaks, respectively, indicate the presence of amide groups in the polymer's side chain as well as main chain and hydroxyl groups in the polymer's side chain. These data was compared with those of the diamine monomer 4 . In the ${ }^{1} \mathrm{H}$ NMR spectrum of diamine monomer 4 , the protons of hydroxyl groups appeared at about $8.60 \mathrm{ppm} .{ }^{32}$ In the ${ }^{1} \mathrm{H}$ NMR spectrum of PAI8b, the resonance of aromatic protons appeared in the range of $6.42-8.29 \mathrm{ppm}$. The proton of the chiral centre appeared as doublet at $4.71-4.72 \mathrm{ppm}$.

\subsection{Organosolubility of PAIs}

It is well-known that the dissolution of polymers depends not only on their physical properties, but also

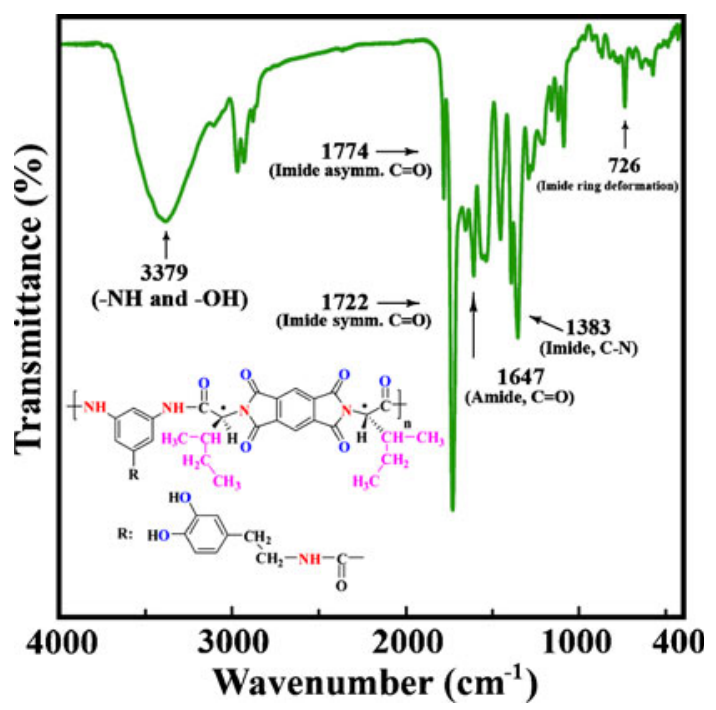

Figure 1. FT-IR spectrum of PAI8b. on their chemical structure. Thus, the introduction of flexible linkages and polar functional groups into the main chain or the side chain of aromatic polymers greatly enhances molecular mobility and provides better solubility. One of the main objectives of this study was producing modified PAIs with improved solubility. Due to the presence of bulky groups as well as hydroxyl functional groups in the polymer side chain, these PAIs have good solubility in different organic solvents. The solubility of obtained PAIs $(\mathbf{8 a}-\mathbf{8 d})$ was investigated as $0.01 \mathrm{~g}$ of polymeric sample in $2 \mathrm{~mL}$ solvent. All of the polymers are soluble in organic solvents such as DMF, DMAc, DMSO, NMP, pyridine, and in $\mathrm{H}_{2} \mathrm{SO}_{4}$ at room temperature and phenolic solvents such as $m$-cresol, and are insoluble in solvents such as methanol, ethanol, acetone, chloroform, methylene chloride, and water. The polymer solutions are very stable, and no gelation, phase separation, or precipitation was observed after storage for several weeks.

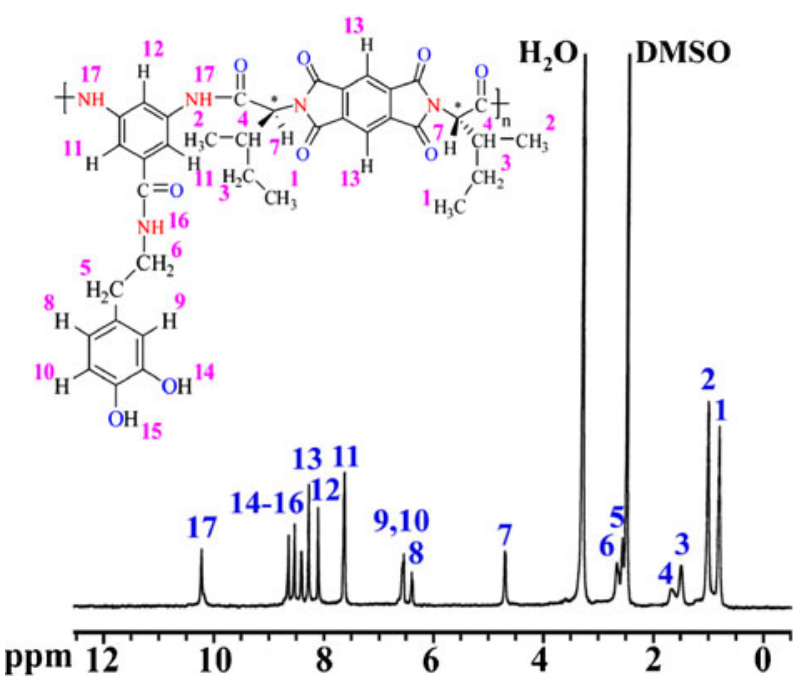

Figure 2. ${ }^{1} \mathrm{H}$ NMR $(400 \mathrm{MHz})$ spectrum of PAI8b in DMSO- $d_{6}$ at RT. 
Table 2. Thermal properties of PAIs $(\mathbf{8 b})$ and $(\mathbf{8 c})^{\mathrm{a}}$.

\begin{tabular}{|c|c|c|c|c|c|}
\hline Polymer & $\mathrm{T}_{5}^{\mathrm{a}}\left({ }^{\circ} \mathrm{C}\right)$ & $\mathrm{T}_{10}^{\mathrm{a}}\left({ }^{\circ} \mathrm{C}\right)$ & Char yield $(\%)^{\mathrm{b}}$ & LOI $(\%)^{\mathrm{c}}$ & $\Delta H_{\text {comb }}(\mathrm{kJ} / \mathrm{g})$ \\
\hline PAI6b & 325 & 376 & 42.2 & 34.4 & 23.2 \\
\hline PAI6c & 332 & 405 & 45.1 & 35.5 & 22.5 \\
\hline
\end{tabular}

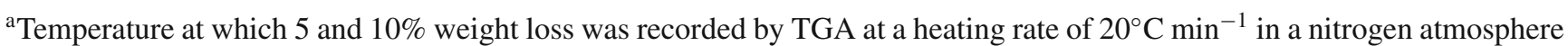
${ }^{b}$ Percentage weight of material left undecomposed after TGA analysis at maximum temperature $800^{\circ} \mathrm{C}$ in a nitrogen atmosphere

${ }^{\mathrm{c}}$ Limiting oxygen index (LOI) evaluating at char yield at $800^{\circ} \mathrm{C}$

\subsection{Thermal stability of PAIs}

The thermal properties of two PAIs $(\mathbf{8 b})$ and $(\mathbf{8 c})$ were examined by TGA and derivative of thermaogravimetric (DTG) in a nitrogen atmosphere at a heating rate of $20^{\circ} \mathrm{C} \mathrm{min}^{-1}$ and the thermal behaviour data are summarized in table 2. Typical TGA curves of representative PAIs $\mathbf{8 b}$ and $\mathbf{8 c}$ in nitrogen atmospheres are shown in figure 3 . The thermal stability of the polymers was studied on the basis of 5 and $10 \%$ weight losses $\left(\mathrm{T}_{5}\right.$ and $\mathrm{T}_{10}$, respectively) of the polymers and the residue at $800^{\circ} \mathrm{C}$. These polymers exhibit good resistance to thermal decomposition, up to $325^{\circ} \mathrm{C}$ in nitrogen, and began to decompose gradually above that temperature. The good thermal stability of resulting polymers is due to the introduction of high rigid pyromellitoyl group into the PAI backbone. $\mathrm{T}_{5}$ for these polymers ranged from 325 to $332^{\circ} \mathrm{C}$ and the $10 \%$ weight loss temperatures of these polymers in nitrogen were recorded in the range of $376-405^{\circ} \mathrm{C}$. The concentration of carbonized residue (char yield) of these polymers in a nitrogen atmosphere was more than $40 \%$ at $800^{\circ} \mathrm{C}$. The high char yields of these polymers can be ascribed to their high aromatic content. The char yield can be applied as a decisive

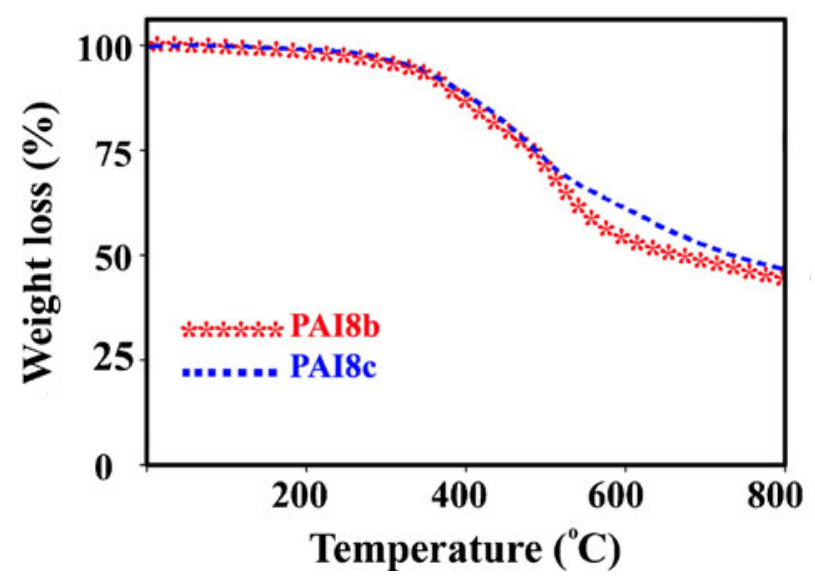

Figure 3. TGA thermograms of PAIs $(\mathbf{8 b})$ and $(\mathbf{8 c})$. factor for estimating the limiting oxygen index (LOI) of polymers according to Van Krevelen equation: ${ }^{37}$

$$
\mathrm{LOI}=17.5+0.4 \mathrm{CR} \quad(\text { where } \mathrm{CR}=\text { Char yield }) .
$$

PAIs $\mathbf{8 b}$ and $\mathbf{8 c}$ had LOI values around 42, which were calculated from their char yield. A polymer having LOI more than 28 is considered as effective flame retardant.

There is also an interesting relationship between the LOI and heat of combustion according to Johnson equation: ${ }^{38}$

$$
L O I=\frac{8000}{\Delta H_{c o m b}},
$$

where $\Delta H_{\text {comb }}$ is the specific heat of combustion in $\mathrm{J} / \mathrm{g}$. So, in the case of these polymers (PAI8b and PAI8c), $\Delta H_{\text {comb }}$ is 23.2 and 22.5 , respectively.

\subsection{XRD analysis}

XRD scans with $2 \theta$ ranging from $10^{\circ}$ to $80^{\circ}$ for powder specimens were obtained for the synthesized PAIs $(\mathbf{8 b})$ and $(\mathbf{8 c})$. As indicated by X-ray diffractograms of

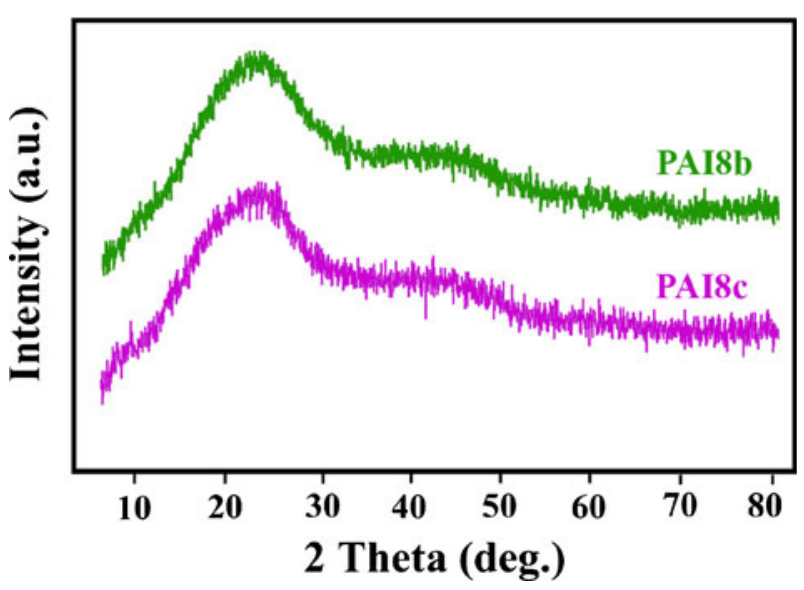

Figure 4. XRD patterns of PAI8b and PAI8c. 


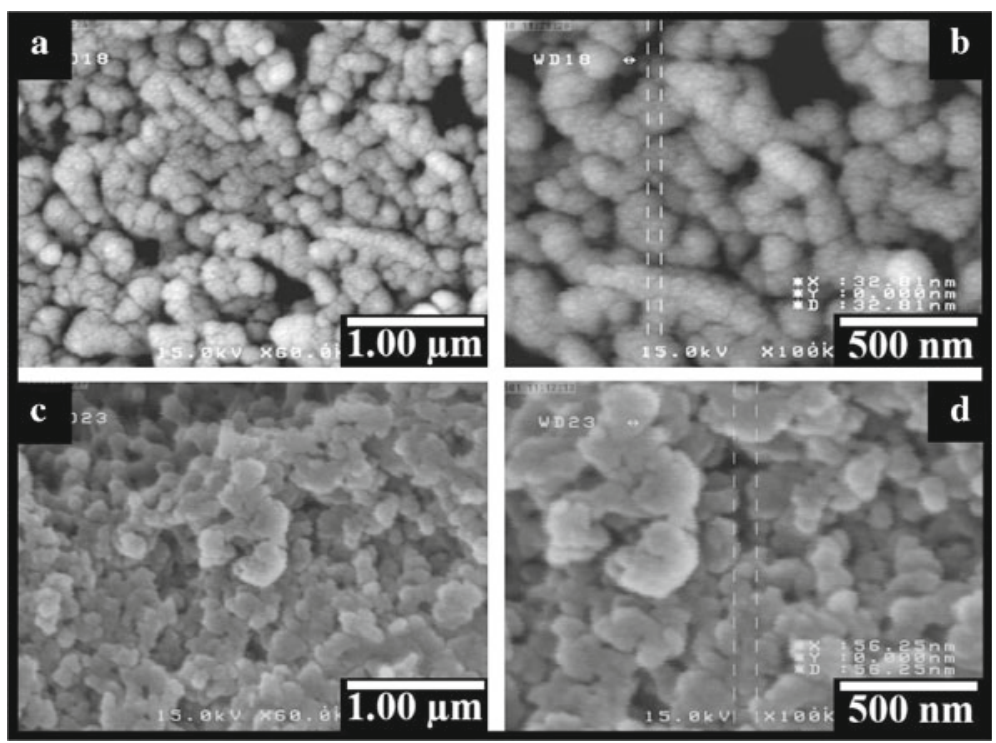

Figure 5. FE-SEM micrographs of PAI8b (a and b) and PAI8c (c and $\mathbf{d})$.

these polymers in figure 4 , the weak reflection centred at a $2 \theta$ value around $20^{\circ}$ was characteristic of the amorphous polymers. This is because the presence of heterocyclic imide groups and aromatic structures in the polymer chain which reduce intermolecular forces between the polymer chains, causing a decrease in crystallinity of all the polymers. On the whole, new wholly aromatic PAIs showed amorphous behaviour.

\subsection{Morphological studies}

Morphological characterization of PAIs was studied by FE-SEM. Shown in figure 5 are the respective
FE-SEM images of PAI8b and PAI8c. FE-SEM observation revealed that PAIs self-organized into nanopatterns. As can be seen from these images, the average diameter of polymeric particles is in the range of $32-56 \mathrm{~nm}$, shape of them is spherical, and they are distributed uniformly and randomly.

It is well-known that ultrasounic irradiation is a well-established method for particle size reduction in dispersions and emulsions as well as generation and application of nano-size materials, because of the potential in the deagglomeration and the reduction of primaries. As most nanomaterials are still fairly expensive, this aspect is of high importance for the commercialization of product formulations containing

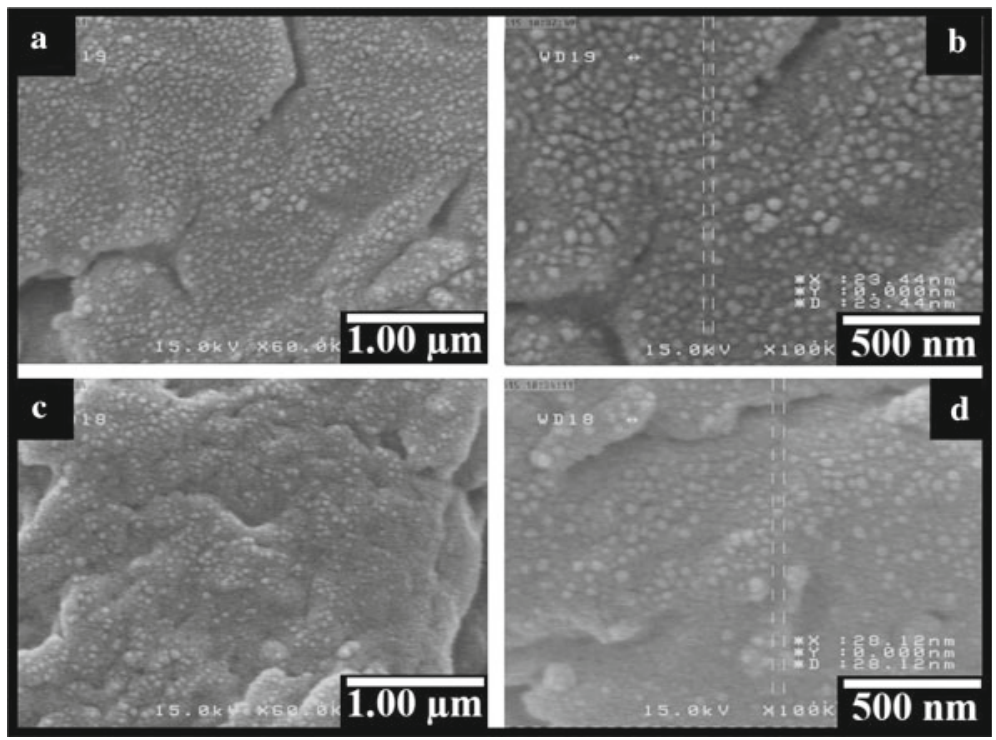

Figure 6. FE-SEM micrographs of PAI8b (a and $\mathbf{b})$ and PAI8c ( $\mathbf{c}$ and $\mathbf{d})$ after sonication process. 
nanomaterials. ${ }^{39}$ Here, powders of polymers have been subjected to irradiation with high-intensity ultrasound for one hour while suspended in ethanol. The homogeneous suspension was placed in a $60^{\circ} \mathrm{C}$ oven overnight to evaporate most of the solvent. Then the semidried polymer powder was further dried in vacuo at $80^{\circ} \mathrm{C}$ for $8 \mathrm{~h}$. The resulting images from FE-SEM confirmed that after ultrasonic irradiation the size of polymeric nanoparticles was decreased (23-28 nm) (figure 6).

\section{Conclusions}

The present study involved the design and synthesis of new optically active nanostructured polymeric materials from the solution polycondensation reaction of 3,5diamino-N-(3,4-dihydroxy-phen-ethyl)benzamide with four $\mathrm{N}, \mathrm{N}^{\prime}$-(pyromellitoyl)-bis-L- $\alpha$-amino acids (8) in molten TBAB as an efficient medium and in the presence of TPP as an activating agent. The reaction conditions open an important alternative to the use of volatile organic solvents. This study shows that we can simply replace the molten ionic salts with traditional volatile and flammable organic solvents in polycondensation reaction between a diamine containing hydroxyl groups and diacids without any reaction of hydroxyl group with acid. The main advantage of this technique is that molten TBAB act as a simple, cheap, safe and excellent medium with a high solubilizing power for the polymerization reactions that increase chemical reactivity and thus lead to better results in comparison with conventional solvents. The easy work-up procedures and recyclability of this molten salt used as the reaction medium makes the method amenable for scale-up operations. Because the resulting polymers had optically pure L-amino acid moieties, they showed optical rotations and were optically active. The presented results also clearly reveal that incorporating the imide group into the polymer main chain as well as combination of the wholly aromatic backbone and several functional groups especially hydroxyl groups remarkably enhanced the thermal stability and solubility of the new polymers and a nice balance of these properties (thermal stability versus solubility) was observed for these polymers. Morphology study of resulting polymers by FE-SEM showed that the polymeric particles are nanosized. The effect of ultrasonic intensity on the PAIs particle size was also studied. FE-SEM images showed that size of particles was decreased. Thermal stability, optical activity, and organosolubility of these polymers make them potential candidates for future high performance applications in high-powered materials, processable high-performance engineering plastics, constructing chiral media for asymmetric synthesis, CSPs for resolution of racemic mixtures by chromatographic techniques, and in related fields.

\section{Acknowledgements}

The authors gratefully acknowledge the financial support from the Research Affairs Division, Isfahan University of Technology (IUT). The financial support from National Elite Foundation (NEF) and Center of Excellence in Sensors and Green Chemistry Research (IUT) is also gratefully acknowledged.

\section{References}

1. Chanda M 2008 Industrial polymers, specialty polymers, and their applications, Salil K Roy (ed) (Kolkata West Bengal, India: CRC Press)

2. Huang L, Peng Z, Guo Y and Porter A L, 2010 J. Bus. Chem. 715

3. Reis C P, Neufeld R J, Ribeiro A J and Veiga F 2006 Nanomedicine 28

4. Mohanraj V J and Chen Y 2006 Trop. J. Pharm. Res. 51

5. Muccini M, Murgia M and Biscarini F 2001 Adv. Mater. 13355

6. Biederman H 2000 RF Vacuum 59594

7. Maurer R 1992 J. Mater. Sci. 276279

8. Rogers R D, Seddon K R (eds) 2003 Ionic liquids as green solvents: Progress and prospects (Washington, DC, USA: ACS Symposium Series)

9. Kim D H, Im J K, Kim D W, Cheong M, Kim H S and Mukherjee D K 2011 J. Chem. Sci. 123467

10. Dupont J, de Souza R F and Suarez P A Z 2002 Chem. Rev. 1023667

11. Ranu B C, Das A and Samanta S 2002 J. Chem. Soc., Perkin Trans. 11520

12. Liu Z, Zhang G, Sun H, Jiang H, Zhao C, Xu D, Li H, Sun X and Na H 2012 Polym. Int. 61565

13. Kissane M, McNamara O A, Mitchell D, Coppert D M, Moynihan H A, Lorenz K T and Maguire A R 2012 Tetrahedron Lett. $\mathbf{5 3} 403$

14. Nagarapu L, Cheemalapati V N, Karnakanti S and Bantu R 2010 Synthesis 193374

15. Mallakpour S and Rafiee Z 2007 Polymer 485530

16. Mallakpour S and Rafiee Z 2007 Eur. Polym. J. 431510

17. Mallakpour S and Zadehnazari A 2010 High. Perform. Polym. 22567

18. Ikai $\mathrm{T}$ and Okamoto Y 2010 In Chiral recognition in separation methods: Mechanisms and applications, A Berthod (ed) (London, New York: Springer Heidelberg Dordrecht) p. 34

19. Okamoto Y, Haruhiko M, Motoshi I, Koichi H, Heimei Y 1986 J. Polym. Sci. Part C, Polym. Symp. 74125

20. Nakano T 2001 J. Chromatogr. A 906205

21. Chen Z, Takei Y, Deore B A and Nagaoka T 2000 Analyst 1252249

22. Petrov V M and Gagulin V V 2001 Inorg. Mater. 32135

23. Ulbricht M 2006 Polymer 472217

24. Shibaev V, Bobrovsky A, Boiko N 2003 Prog. Polym. Sci. 28729 
25. Singer K D, Petschek R G, Ostroverkhov V and Twieg R J 2003 J. Polym. Sci., Part B: Polym. Phys. 41 2744

26. Kauranen M, Verbiest T, Maid J J and Persoons A 1996 Synth. Met. 81117

27. Okamoto Y 2009 J. Polym. Sci., Part A: Polym. Chem. 471731

28. Liu R, Sanda F and Masuda T 2008 J. Polym. Sci., Part A: Polym. Chem. 464175

29. Miyabe T, Iida H, Banno M, Yamaguchi T and Yashima E 2011 Macromolecules 448687

30. Inoue M, Teraguchi M, Aoki T, Hadano S, Namikoshi T, Marwanta E and Kaneko T 2009 Synth. Met. 159854

31. Mallakpour S and Zadehnazari A 2011 eXPRESS Polym. Lett. 5142
32. Mallakpour S, Hatami M, Ensafi A A and Karimi-Maleh H 2011 Chin. Chem. Lett. 22185

33. Mallakpour S E, Hajipour A R and Habibi S 2001 Eur. Polym. J. 372435

34. Mallakpour S and Shahmohammadi M H 2004 J. Appl. Polym. Sci. 92951

35. Mallakpour S and Shahmohammadi M H 2005 Iran. Polym. J. 14473

36. Faghihi K, Foroughifar N and Mallakpour S 2004 Iran. Polym. J. 1393

37. Van Krevelen D W 1975 Polymer 16615

38. Johnson P R 1974 J. Appl. Polym. Sci. 18491

39. Mason T J and Lorimer J P 2002 Applied sonochemistry: Uses of power ultrasound in chemistry and processing (Verlag GmbH and Co. KGaA: Wiley-VCH) 